\title{
Acceptance and Completion of Rifapentine-based TB Preventive Therapy (3HP) Among People Living with HIV (PLHIV) in Kampala, Uganda - Patient and Health Worker Perspectives.
}

\section{Fred C. Semitala ( $\square$ semitala@gmail.com )}

Makerere University College of Health Sciences https://orcid.org/0000-0002-0624-7640

Allan Musinguzi

Infectious Diseases Research Collaboration

Jackie Ssemata

Infectious Diseases Research Collaboration, Kampala, Uganda

Fred Welishe

Infectious Diseases Research Collaboration, Kampala, Uganda

Juliet Nabunje

Infectious Diseases Research Collaboration, Kampala, Uganda

\section{Jillian L. Kadota}

Division of Pulmonary and Critical Care Medicine and Center for Tuberculosis, University of California San Francisco, San Francisco CA USA

\section{Christopher A. Berger}

Division of Pulmonary and Critical Care Medicine and Center for Tuberculosis, University of California San Francisco, San Francisco CA USA

\section{Achilles Katamba}

School of Medicine, Makerere University College of Health Sciences, Kampala, Uganda; Uganda

Tuberculosis Implementation Research Consortium, Kampala, Uganda

\section{Noah Kiwanuka}

School of Public Health, Makerere University College of Health Sciences, Kampala, Uganda

\section{Moses R. Kamya}

School of Medicine, Makerere University College of Health Sciences, Kampala, Uganda; Infectious

Diseases Research Collaboration, Kampala, Uganda

\section{David Dowdy}

Department of Epidemiology, Johns Hopkins Bloomberg School of Public Health, Baltimore, MD USA; Uganda Implementation Research Consortium, Kampala, Uganda

\section{Adithya Cattamanchi}

Division of Pulmonary and Critical Care Medicine and Center for Tuberculosis, University of California San Francisco, San Francisco CA USA; Uganda Tuberculosis Implementation Research Consortium, 
Kampala, Uganda

Anne R. Katahoire

Child Health and Development Centre, Makerere University College of Health Sciences, Kampala, Uganda

\section{Research}

Keywords: tuberculosis, preventive therapy, perceived barriers, perceived facilitators, people living with HIV, health workers, implementation, qualitative methods

Posted Date: October 19th, 2020

DOI: https://doi.org/10.21203/rs.3.rs-92783/v1

License: (c) (1) This work is licensed under a Creative Commons Attribution 4.0 International License. Read Full License

Version of Record: A version of this preprint was published at Implementation Science Communications on June 30th, 2021. See the published version at https://doi.org/10.1186/s43058-021-00173-2. 


\section{Abstract}

Background: A 12-dose, once-weekly regimen of isoniazid and rifapentine (3HP) is effective in preventing tuberculosis (TB) among people living with HIV (PLHIV). We sought to identify potential barriers to and facilitators of $3 \mathrm{HP}$ implementation from the perspective of PLHIV and health workers in a routine HIV care setting in Kampala, Uganda.

Methods: We conducted semi-structured interviews with 25 PLHIV and 10 health workers at an HIV/AIDS clinic in Kampala, Uganda. For both groups, we explored their understanding and interpretations of TB and TB preventive therapy (TPT), and perceptions about social and contextual factors that might influence willingness of PLHIV to initiate and complete 3HP. We analyzed the data using an inductive thematic approach and aligned the emergent themes to the Behavior Change Wheel framework to identify sources of behavior and targeted behavior change interventions.

Results: Facilitators for uptake and completion of 3HP among PLHIV were fear of contracting TB, awareness of being potentially at risk of getting TB, willingness to take TPT, trust in health workers, and the perceived benefits of DOT and SAT. Barriers included inadequate understanding of TPT, fear of potential side effects, concerns about effectiveness of 3HP as well as the perceived challenges of DOT or SAT. Among health workers, perceived facilitators included knowledge that TB is a common cause of mortality, fear of getting TB, patient trust in their health workers, potential for once-weekly dosing and the benefits of TPT delivery. Health workers perceived potential barriers for PLHIV as being: inadequate understanding of TB and TPT, TB associated stigma, potential side effects, pill burden and challenges of taking TPT. Additional barriers included a lack of experience among health workers with the use of digital technology to monitor patient care.

Conclusions: Using a formative qualitative and comprehensive theoretical approach, we identified key sources of behavior that could be used to guide selection of appropriate intervention to optimize $3 \mathrm{HP}$ scale up among PLHIV in high burden settings.

\section{Contributions To The Literature}

- Newer, shorter and safer regimens for TB prevention are becoming more available. However, their adoption into national TB/HIV programs has been slow in low-income settings.

- The findings from this study elaborate the key facilitators of and barriers to 3HP uptake as perceived by PLHIV and health workers in a low-income setting.

- Stakeholder perspectives can help guide development of interventions to support 3HP scale-up in similar settings.

\section{Background}

Tuberculosis (TB) is the leading cause of death among people living with HIV (PLHIV) worldwide[1]. TB preventive therapy (TPT) is recommended by the World Health Organization (WHO) for all PLHIV without 
active TB [2-4]. The traditional TPT regimen has involved patients taking six to nine months of daily isoniazid [5]. However, initiation and completion of daily isoniazid treatment among PLHIV has been poor worldwide due to concerns about toxicity and the long duration of treatment $[6,7]$.

As an alternative, in 2018, WHO recommended a three-month (12-dose) regimen of weekly isoniazid and rifapentine (3HP) based on randomized trials showing equivalent efficacy, better tolerability and higher treatment completion compared to six months of daily isoniazid[4, 8, 9]. In these studies, each weekly dose of 3HP was administered to PLHIV under direct observation of a health worker (directly observed therapy, DOT) to ensure adherence and provide opportunity for monitoring and managing adverse events. However, DOT may pose challenges to acceptance and scale-up of 3HP among PLHIV and health workers in low-income countries. These challenges include direct and indirect costs of attending weekly clinic visits consuming up to $40 \%$ of median personal weekly income [10], lack of time to attend clinic visits and increased workload for health workers. While self-administered therapy (SAT) overcomes some of these challenges, it could lead to delayed recognition of adverse events and lower adherence in some contexts[11].

Formative studies help researchers to optimize the design and implementation of evidence-based interventions by tailoring them to fit within the real-world practice contexts of key stakeholders [12-15]. We conducted a qualitative study to identify potential barriers to, and facilitators of, 3HP acceptance and completion, from the perspective of PLHIV in HIV/AIDS care and health workers at a large, urban HIV/AIDS clinic in Kampala, Uganda. The goal was to inform the design of SAT and DOT delivery strategies for a type 3 hybrid effectiveness-implementation trial of 3HP [16].

\section{Methods}

\section{Study design and population sample}

We conducted semi-structured interviews between January and March 2019 among PLHIV and health workers at the Mulago Immune Suppressive Syndrome (i.e., AIDS) clinic. The Mulago AIDS clinic provides comprehensive HIV/TB care and treatment services to over 16,000 PLHIV, largely from Kampala (the capital city of Uganda) and surrounding districts. We purposively sampled consecutive PLHIV engaged in care at the clinic during their routine appointments. We also purposively sampled full-time health workers who were involved in the provision of TB treatment and prevention services at the clinic. Interviews with PLHIV and health workers lasted between 25 and 40 minutes. Informed verbal consent was obtained from all participants.

The study received ethical approvals from the School of Public Health Higher Degrees Research and Ethics Committee at the Makerere University College of Health Sciences, the Uganda National Council of Science and Technology and the University of California San Francisco Committee on Human Research.

\section{Recruitment of study participants}


All adult ( $\geq 18$ years old) PLHIV engaged in care at the clinic who were able to provide informed verbal consent in English or Luganda (the most-widely spoken local language) were considered for enrollment into the study. PLHIV were recruited by two study nurses from the patient waiting area or were referred by clinic staff at the patient triage area. The study team purposively sampled PLHIV to achieve variation based on the duration of care at the clinic, as well as demographic characteristics such as sex, age, and distance travelled to reach the clinic. Health workers were recruited based on their active involvement in providing TB-related care at the clinic.

\section{Study instruments and data collection}

Separate interview guides were developed for PLHIV and health workers. Both interview guides included open-ended questions designed to explore potential barriers to and facilitators of the acceptance and completion of 3HP by PLHIV at the Mulago AIDS clinic.

Interview guides for PLHIV explored their understanding of TB (of the disease itself, transmission dynamics, prevention and treatment), their perceptions of individual risk of contracting TB disease and awareness about TPT and their perception of its importance. Other questions were specific to 3HP and explored willingness to take 3HP if it were offered to them by their health worker, how easy/difficult it would be to remember to take the once-weekly dose, perceived advantages or disadvantages of either taking 3HP via DOT or SAT, and perceptions about use of digital adherence technologies to support 3HP adherence and completion.

Interview guides for health workers included questions about their experiences with PLHIV at the clinic, experiences with TB and TPT and their perceptions about concerns PLHIV may have about TB and TPT. The guide also explored perceived challenges or benefits that their clients may encounter with DOT vs. SAT, their own preferences for delivering 3HP by DOT vs. SAT, and their perceptions about use of digital adherence technologies to support 3HP completion.

Both interview guides were drafted in English, piloted and refined using a convenience sample of PLHIV and health workers at the clinic. The interview guide for PLHIV was then translated professionally to Luganda and checked for accuracy by members of the local study team who were bilingual.

All interviews were audio-recorded and transcribed verbatim. All Luganda transcripts were then translated to English for analysis.

\section{Analysis}

All transcripts were coded manually and analyzed using inductive thematic analysis $[17,18]$. The themes generated were classified as potential barriers or facilitators to 3HP acceptance and completion. The emergent barriers and facilitators were then mapped to the Capability Opportunity Motivation Behavior (COM-B) model [19]. The Behavior Change Wheel (BCW) framework[20] was used to identify potential interventions to overcome the barriers and promote the facilitators of acceptance and completion of $3 \mathrm{HP}$ by PLHIV. 


\section{Results}

\section{Demographic characteristics of study participants}

Twenty-five (25) PLHIV and 10 health workers participated in the interviews. Of the 25 PLHIV, 16 (64\%) were female, and the median age was 39 years (IQR: 31-45). Their duration in HIV/AIDS care at the clinic ranged from 8 months to 14 years (median 6 years; IQR: 5-10 years). The 10 health workers included three medical doctors, three nurses, two clinical officers, one pharmacist and one pharmacy technician. Six health workers (60\%) were female, and the median age was 29.5 years (IQR: 28-35 years). The duration in service at the current post ranged from 6 months to 11 years (median 2.5 years; IQR: 27 years).

\section{Patient-reported barriers and facilitators}

Almost all PLHIV were aware that their HIV-positive status put them at a higher risk of developing active TB and were willing to take $3 \mathrm{HP}$ to lower their risk. They also understood that TB can easily spread from person to person. Almost all were open to taking medicines if it was recommended by their health workers at the clinic in order to reduce their chances of contracting TB (Table 1).

"TB is a terrible disease; what I know about it is it spoils one of the organs and that is the lungs and once the lungs are spoilt then of course next is death......And I have seen very many people suffering from $T B$ you wouldn't like the same. So, if there is a chance of prevention, I welcome it". (Middle-aged Male, PLHIV interview)

Most PLHIV preferred self-administration of 3HP as it would be cheaper, would suit their daily lives better and would allow them to take their medicines at their convenience.

"You are not inconvenienced. You can even take the medicine and finish it without anyone knowing that you are on medication. Even at work you will not be disturbed by having to seek permission". (Middle-aged Female, PLHIV interview)

At the same time, the delivery of 3HP using the DOT strategy was perceived by PLHIV as potentially beneficial given greater contact with the health workers, who would ensure that they took the medication and could address any patient concerns or side effects.

Several of the PLHIV interviewed were: 1) unaware that TB could be prevented, 2) did not perceive the importance of TB preventive therapy or 3) queried the logic of treating someone who is not "sick" with TB:

"My thoughts are why you give me medicine if I am healthy.... You may find that God did not plan for them to die of the disease-causing organism for TB and yet they are taking medicine... No. I do not support that!" (Young Female, PLHIV interview)

PLHIV perceived the weekly 3HP dose, which consists of 11 tablets, as too many and potentially prohibitive. Some doubted their own ability to swallow 11 tablets at once: 
"The problem is because for me I have been taking 2 tablets [Septrin and ARVs], now what has shocked me is taking 11 tablets. I don't know the danger with taking because I have never taken such many tablets". (Middle-aged Male, PLHIV interview)

PLHIV feared potential side effects, believing that the medicines used to treat TB were "tough" on the body and were concerned that they could arise especially when combined with their current ART regimens. Some of the participants were also concerned about the duration of protection afforded by 3HP:

"My concern might be, leave alone the side effects when you take it aren't you very vulnerable to contract TB again.... So, I don't know, will I be vulnerable to such TB or will I be protected"? (Middle-aged Female, PLHIV interview).

The weekly clinic appointments associated with DOT were perceived as costly in terms of transport, time, childcare, and absence from work. Those who resided far away from the clinic expressed additional concern:

"One challenge would be transport costs will be high.... Transport costs, sometimes time, and maybe getting permission from work". (Young Female, PLHIV interview)

SAT as a delivery strategy was perceived to be convenient but some expressed that the lack of dosing supervision combined with the unusual once-weekly dosing schedule could potentially lead to poor adherence to 3HP:

"Now doctor, some people you can give them medicine and when they come here, and you ask them; did you take your medicine? They will say yes. But truthfully, they got 11 tablets, took three of them and stopped". (Middle-aged Female, PLHIV interview)

\section{Health worker-reported barriers and facilitators}

All health workers interviewed mentioned that TB was the leading cause of severe illness and death among PLHIV in care at the clinic and perceived TB prevention as very important (Table 2).

"In this clinic, of the 16,000 patients, most are stable and if you look at the cause of mortality it is TB related. ... So, it deserves much attention". (Medical Doctor at the clinic)

Almost all the health workers felt that taking 11 tablets at once would be perceived as too high a pill burden, especially for those already struggling to adhere to their daily ART.

"...I have been concentrating on my triple [three daily ARV pills] and now you want to give me more medication. ... some of them are not so positive about it." (Medical Doctor at the clinic).

Yet, health workers believed that the fear of getting TB would motivate PLHIV to take and complete 3HP. They also conceded that the once-weekly dosing schedule of 3HP could convince some PLHIV to take it despite the potential pill burden. 
"Taking it once a week rather than taking it daily much as the tablets are many. Maybe one will be like "anyway am taking many but once a week". (Medical Doctor at the clinic)

Most health workers preferred to treat PLHIV using DOT to ascertain completion of the required doses but acknowledged that most PLHIV in their care would most likely prefer SAT given the implications of costs and convenience. Conflicting work schedules were also perceived as a potential challenge for weekly clinic appointments.

Health workers perceived inadequate knowledge about TB as a potential challenge for PLHIV to accept and complete 3HP. They noted that while most PLHIV knew the common signs and symptoms of TB, they still had misconceptions such as TB being hereditary, or transmitted through cigarette smoking and alcohol drinking. These misconceptions could potentially result in non-acceptance of TPT or poor adherence once initiated on treatment. They also noted that TB is a highly stigmatized disease both at the clinic and within the communities where patients reside. Due to this and drawing from their experiences at the clinic, some health workers believed that PLHIV would be uncomfortable to be seen taking TB medicines. Health workers also observed that PLHIV would be concerned about potential side effects, while others mistrusted any new medications:

"Balese biragala kututta." (This literally means they have brought medications to kill us) ... "And then other people think that the TB prevention medications, are very strong, they are very toxic that they will affect the liver and the kidney". (Nurse at the clinic)

Finally, most health workers reported that they had never used technology to monitor drug adherence of PLHIV remotely and were uncertain about the reliability of adherence determined electronically:

"Technology no, mostly we depend on self-report, pill count, say I have been taking so what is your balance? Then you negotiate around that. Technology no." (Nurse at the clinic)

\section{Behavioral diagnosis and intervention options}

The behavioral diagnosis obtained from the patient and provider reported barriers to acceptance and completion of 3HP encompassed all the six domains of the COM-B model (i.e., physical and psychological capability, physical and social opportunity as well as reflective and automatic motivation), as summarized in Table 3.

By linking the behavioral diagnosis obtained using the COM-B model to the BCW framework, we identified appropriate intervention functions that could be used to modify the behavior of both PLHIV and health workers to facilitate the acceptance and completion of 3HP (Table 4). These intervention functions included modifying the psychological capability of health workers through training on how to use digital adherence technology to monitor adherence of PLHIV to 3HP and solving the psychological capability barriers of PLHIV through education, which could help to address the misconceptions held by PLHIV about TB and TPT. The other intervention functions identified included use of dosing/appointment reminders to enable PLHIV to remember to take their once-weekly 3HP doses, the use of persuasion to 
convince PLHIV to accept $3 \mathrm{HP}$ and restructuring the clinic environment to reduce waiting time when patients present for TPT visits.

\section{Discussion}

The goal of this formative qualitative study was to identify potential barriers to and facilitators of uptake and completion of 3HP TPT, as perceived by PLHIV and health workers, to inform the design of optimized SAT and DOT delivery strategies. The key potential facilitators for uptake and completion of 3HP among PLHIV included fear of contracting TB, trust in health workers, the once-weekly 3HP dosing schedule and the perceived benefits of both DOT and SAT delivery models. Key potential barriers identified were inadequate understanding of TPT, fear of 3HP pill burden, potential side effects, concerns about effectiveness of 3HP and the perceived challenges of DOT or SAT. In addition, health workers also had no prior experience with the use of digital technology to monitor patient care. These findings highlight the need for targeted implementation support to enhance uptake of $3 \mathrm{HP}$ in this setting.

Studies from other high HIV and TB burden countries have shown that ineffective communication between patients and health workers may lead to a misunderstanding of the preventive role of TPT by patients and influences their acceptance of and adherence to TPT [21, 22]. Both the PLHIV and health workers affirmed that patients trust the health workers at the clinic and would be receptive to 3HP if their health worker recommended it. Once patients are convinced about the benefits and delivery models for $3 \mathrm{HP}$, they are well-positioned to guide its scale up. In addition, health workers believed that the onceweekly 3HP dosing schedule could be used as an advantage to encourage PLHIV to accept and complete treatment. Several studies have previously demonstrated that trust in health workers influences patient adherence to and completion of TPT [21-23].

Other perceived challenges associated with 3HP delivery included increased costs of attending more frequent clinic visits, conflicting work schedules and forgetting to take 3HP dosages due to the unfamiliar once-weekly dosing schedule. Other studies have also reported clinic-based DOT, financial barriers, forgetfulness, work duties, childcare responsibilities and other competing priorities as challenges to acceptance and completion of TPT [21,23,24]. As described by Stennis et al, allowing PLHIV to select a mode of delivery for 3HP is likely to increase acceptance and completion [25].

Both PLHIV and health workers were hesitant to promote the use of digital adherence monitoring for PLHIV on SAT since they had no experience in using such technology, and therefore perceived it as unreliable for monitoring patient adherence to medicines. The WHO recommends the integration of digital health technologies into TB prevention and care activities in support of its End TB Strategy[26]. In accordance with this, there is a greater need to empower health workers in resource limited setting with skills to use this technology for better care. In addition to scale-up of health worker training, promoting confidence in the use of digital technology to monitor patient care can be increased by sharing examples of the successful use of technology elsewhere [27]. 
This study provides insight into the perceptions of the individuals (PLHIV and health workers) directly affected by implementation of 3HP, and are critical to scaling up 3HP for prevention of TB $[28,29]$ Identification of barriers to and facilitators of 3HP acceptance and completion informs a better understanding of the contextual factors that could potentially influence intervention implementation and is useful to guide the development and implementation of targeted interventions. To our knowledge, our study is the first to assess potential barriers and facilitators to the implementation of 3HP in sub-Saharan Africa and it highlights key considerations that require attention to support effective scale-up [30].

The strength of our study lies in the application of several core principles of implementation science used in developing complex interventions: 1) identifying an evidence-practice gap, 2) conducting formative research and 3) analyzing results using a validated theory of behavior change.[31] Our interviews included both health care providers and PLHIV, populations which are critical for the scale-up of 3HP-based TB preventive therapy. Importantly, these findings provide a perspective of a wide spectrum of PLHIV who have been in care for varying durations ranging from less than one year to 14 years.

Our study has some limitations. First, we collected data at a single health facility which is well resourced for HIV service delivery. The barriers and facilitators may be different from other clinical contexts. Second, although these perspectives were obtained from a wide spectrum of PLHIV receiving care at the clinic, including a balanced gender representation, it is possible that the results may be different with varying cultural contexts. Third, there might be other barriers to 3HP that go beyond the purview of patients and health workers that we were not able to study. However, by combining patient and health worker perspectives of patients, these findings highlight key issues that need to be addressed while considering scale up of 3HP-based TPT in high HIV/TB burden settings such as Uganda and beyond.

\section{Conclusions}

Using a formative qualitative and comprehensive theoretical approach, we identified key sources of behavior and appropriate intervention functions that could be used to optimize the delivery of $3 \mathrm{HP}$ to PLHIV in our setting. In addition, we also laid a strong foundation for the development and implementation of 3HP in other high HIV/TB burden settings where similar barriers and facilitators are likely to exist.

\section{Abbreviations}

3HP: Combination of Isoniazid and Rifapentine medicines taken once-weekly for 12 weeks

PLHIV: People living with HIV

TB: Tuberculosis

HIV: Human Immunodeficiency Virus

AIDS: Acquired Immunodeficiency Syndrome 
TPT: Tuberculosis Preventive Therapy

COM-B: Capability Opportunity Motivation Behavior

BCW: Behavior Change Wheel framework

SAT: Self-Administered Therapy

DOT: Directly Observed Therapy

WHO: World Health Organization

IPT: Isoniazid Preventive Therapy

SMS: Short Message Service

LTBI: Latent Tuberculosis Infection

\section{Declarations}

\section{Ethics approval and consent to participate:}

This study was approved by the School of Public Health Higher Degrees Research and Ethics Committee at the Makerere University College of Health Sciences, the Uganda National Council of Science and Technology and the University of California San Francisco Committee on Human Research. All participants provided informed consent.

\section{Consent for publication:}

Not applicable

\section{Availability of data and materials:}

The datasets used and/or analyzed during the current study are available from the corresponding author on reasonable request.

\section{Competing interests:}

The authors declare that they have no competing interests

\section{Funding:}

This study was supported by a grant from the U.S. National Heart, Lung and Blood Institute: NIH/NHLBI R01HL144406. Dr. Semitala's training in implementation science was supported by the Fogarty International Center of the National Institutes of Health under Award Number D43 TW010037. The funders 
had no role in the design, collection, analysis, and interpretation of data; in the writing of the manuscript; and in the decision to submit this manuscript for publication.

\section{Author's contributions:}

FCS, DW and AC designed the study. AM, ARK, AK, NK, MRK and FCS oversaw the local collection of data. JS, FW and JN collected the data. FCS, AM and AK analyzed the data and wrote the manuscript with input from all authors. AC, DW, JLK and AK critically reviewed the manuscript. All authors read and approved the final manuscript.

\section{Acknowledgements:}

The authors are grateful to the administration, staff, and patients at the Makerere Joint AIDS Program Mulago (ISS) HIV/AIDS clinic for their time and participation. We also thank the Infectious Diseases Research Collaboration, the Uganda TB Implementation Research Consortium (U-TIRC), and the Uganda National Tuberculosis and Leprosy Program for supporting the Study.

\section{References}

1. UNAIDS. Tuberculosis - good progress, but not enough. [Online] 202023 March 2020 [cited 20208 May 2020]; Available from: https://www.unaids.org/en/resources/presscentre/featurestories/2020/march/20200323_tb.

2. Badje, A., et al., Effect of isoniazid preventive therapy on risk of death in west African, HIV-infected adults with high CD4 cell counts: long-term follow-up of the Temprano ANRS 12136 trial. 2017. 5(11): p. e1080-e1089.

3. Medicine, T.A.S.G.J.N.E.J.o., A trial of early antiretrovirals and isoniazid preventive therapy in Africa. 2015. 373(9): p. 808-822.

4. Organization, W.H., Latent tuberculosis infection: updated and consolidated guidelines for programmatic management. 2018, World Health Organization.

5. WHO and W.H. Organization, Guidelines on the management of latent tuberculosis infection. 2015: World Health Organization.

6. Namuwenge, P., et al., Loss to follow up from isoniazid preventive therapy among adults attending HIV voluntary counseling and testing sites in Uganda. 2012. 106(2): p. 84-89.

7. Organization, W.H., Report of a" lessons learnt" workshop on the six ProTEST pilot projects in Malawi, South Africa and Zambia. 2004, World Health Organization.

8. Sterling, T.R., et al., Three months of rifapentine and isoniazid for latent tuberculosis infection. 2011. 365(23): p. 2155-2166.

9. Martinson, N.A., et al., New regimens to prevent tuberculosis in adults with HIV infection. 2011. 365(1): p. 11-20. 
10. Kadota, J.L., et al., Willingness to accept reimbursement for visits to an HIV clinic for tuberculosis preventive therapy. Int J Tuberc Lung Dis, 2020. 24(7): p. 729-731.

11. Belknap, R., et al., Self-administered Versus Directly Observed Once-Weekly Isoniazid and Rifapentine Treatment of Latent Tuberculosis Infection: A Randomized Trial. Ann Intern Med, 2017. 167(10): p. 689-697.

12. Aakhus, E., et al., Tailoring interventions to implement recommendations for the treatment of elderly patients with depression: a qualitative study. 2015. 9(1): p. 36.

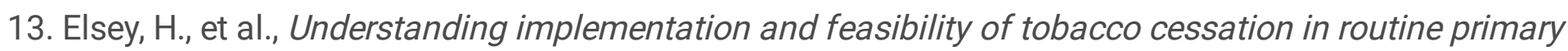
care in Nepal: a mixed methods study. 2015. 11(1): p. 104.

14. Reger, G.M., et al., Barriers and facilitators to mobile application use during PTSD treatment: Clinician adoption of PE coach. 2017. 48(6): p. 510.

15. Health, U.D.o. and M. Human Services \%J National Institutes of Health. Bethesda, USA: National Cancer Institute, Qualitative methods in implementation science. 2018.

16. Kadota, J.L., et al., Protocol for the 3HP Options Trial: a hybrid type 3 implementation-effectiveness randomized trial of delivery strategies for short-course tuberculosis preventive therapy among people living with HIV in Uganda. Implement Sci, 2020. 15(1): p. 65.

17. Javadi, M. and K.J.D. Zarea, Understanding thematic analysis and its pitfall. 2016. 1(1): p. $33-39$.

18. Boyatzis, R.E., Transforming qualitative information: Thematic analysis and code development. 1998: sage.

19. Mayne, J.J.u., www. researchgate. net/publication/314086441_The_COM-B_Theory_of_ Change_Model_V3, The COM-B theory of change model. 2018.

20. Michie, S., M.M. van Stralen, and R. West, The behaviour change wheel: a new method for characterising and designing behaviour change interventions. Implement Sci, 2011. 6: p. 42.

21. Mindachew, M., et al., Perceived barriers to the implementation of Isoniazid preventive therapy for people living with HIV in resource constrained settings: a qualitative study. 2014. 17(1).

22. Jacobson, K.B., et al., "It's about my life": facilitators of and barriers to isoniazid preventive therapy completion among people living with HIV in rural South Africa. 2017. 29(7): p. 936-942.

23. Kim, H., et al., Priorities among HIV-positive individuals for tuberculosis preventive therapies. 2020. 24(4): p. 396-402.

24. Stennis, N.L., et al., Treatment for tuberculosis infection with 3 months of isoniazid and rifapentine in New York City health department clinics. 2016. 62(1): p. 53-59.

25. Stennis, N.L., et al., Treatment for Tuberculosis Infection With 3 Months of Isoniazid and Rifapentine in New York City Health Department Clinics. Clin Infect Dis, 2016. 62(1): p. 53-59.

26. Organization, W.H., Digital health for the End TB Strategy: an agenda for action. 2015, World Health Organization.

27. Lam, C.K. and K. McGinnis Pilote, Using Video Technology to Increase Treatment Completion for Patients With Latent Tuberculosis Infection on 3-Month Isoniazid and Rifapentine: An Implementation 
Study. 2018. 20(11): p. e287.

28. Mindachew, M., et al., Perceived barriers to the implementation of Isoniazid preventive therapy for people living with HIV in resource constrained settings: a qualitative study. Pan Afr Med J, 2014. 17: p. 26.

29. Pease, C., et al., Efficacy and completion rates of rifapentine and isoniazid (3HP) compared to other treatment regimens for latent tuberculosis infection: a systematic review with network meta-analyses. BMC Infect Dis, 2017. 17(1): p. 265.

30. Jillian L. Kadota, A.M., Juliet Nabunje, Fred Welishe, Jackie L. Ssemata, Opira Bishop, Christopher A. Berger, Devika Patel, Amanda Sammann, Anne Katahoire, Payam Nahid, Robert Belknap, Patrick PJ Phillips, Jennifer Namusobya, Moses Kamya, Margaret A. Handley, Noah Kiwanuka, Achilles Katamba, David Dowdy, Fred C. Semitala, Adithya Cattamanchi, Protocol for the 3HP Options Trial: a hybrid Type 3 implementation-effectiveness randomized trial of delivery strategies for short-course tuberculosis preventive therapy among people living with HIV in Uganda. . In Press, implementation Scienece, 2020.

31. Craig, P., et al., Developing and evaluating complex interventions: the new Medical Research Council guidance. 2008. 337: p. a1655.

\section{Tables}


Table 1

Patient-reported barriers to and facilitators of use, completion and models of delivery for 3HP at Mulago ISS clinic in Uganda

\section{Potential Facilitators}

Fear of contracting TB - TB is a terrible disease what I know about it is it spoils one of the organs and that is the lungs and once the lungs are spoilt then of course next is death......And I have seen very many people suffering from TB you wouldn't like the same. So, if there is a chance of prevention, I welcome it. (Middle-aged Male, PLHIV interview)

Aware of being potentially at risk of TBYes, mostly people who have HIV it is so good because our bodies are not strong it gets sick easily but if I prevent it means it is not easy to get. (Young Female, PLHIV interview)

\section{Awareness that TB is easily} transmissible- What I know is that when someone is suffering from it, there is a high chance when you are sitting with that one, when you are sharing cups, when she is a wife and you are sleeping together, when you are a parent and your children are there, of course there is a lot of communication. So, it is terrible if you have a home, the whole home may be affected. (Middle-aged Male, PLHIV interview)

\section{Willingness to take TB preventive} therapy- Eeh! My dear, they say prevention is better than cure. That is why I am here. You never know you can get that disease and you get problems treating it. But if you prevent against it and you do not get it, it's better. (Middleaged Male, PL HIV interview)

Trust in health workers -No, I don't see any problem what I have realized with experience is that the medical people can't recommend something that can be harmful. (Middle-aged Male, PLHIV interview)

\section{Potential Barriers}

Inadequate understanding of TPT - My thoughts are why do you give me medicine if I am healthy.... You may find that God did not plan for them to die of the diseasecausing organism for TB and yet they are taking medicine... No. I do not support that! (Young Female, PLHIV interview)
Potential Pill burden - The problem is because for me I have been taking 2 tablets (Septrin and ARVs), now what has shocked me is taking 11 tablets. I don't know the danger with taking because I have never taken such many tablets. (Middle-aged Male, PLHIV interview)

Fear of potential side effects - The question I can ask about is whether it can have side effects. Those side effects are the ones we fear the most about medicine because now we see prevention but if I started it what would I look like? What would it make me look like? How would it treat me? All those things have to be known because you can start taking it and you become very strange. The eyes change, and you get to a point where you cannot do your work to earn a living. Yet also if you are taking medicine but are not feeding well you may not stay long. (Young Female, PLHIV interview)

Concerns about effectiveness of 3HP- My concern might be, leave alone the side effects when you take it aren't you very vulnerable to contract TB again.... So, I don't know, will I be vulnerable to such TB or I will be protected? (Middle-aged Female, PLHIV interview)
Perceived challenges of DOT -One challenge would be transport costs will be high.... Transport costs, sometimes time, and maybe getting permission from work. (Young Female, PL HIV interview) 


\section{Potential Facilitators}

Perceived benefits of DOT - Because they take under your observation and you make sure that they have taken it. You know that they have taken it but this person taking from home is on probability. They may take or may not take, and you would not know. But for the person here you would be on sure deal because you have observed them taking it. (Middle-aged Female, PLHIV interview)

Perceived benefits of SAT - You are not inconvenienced. You can even take the medicine and finish it without anyone knowing that you are on medication. Even at work you will not be disturbed by having to seek permission. (Middle-aged Female, PLHIV interview)

\section{Potential Barriers}

Perceived challenges of SAT- Now doctor, some people you can give them medicine and when they come here, and you ask them; did you take your medicine? They will say yes. But truthfully, they got 11 tablets, took three of them and stopped. And yet, you told them, you take all 11 tablets at once. They will take only two tablets and the rest, they fail. If they get time like the following day, then they take again. Doesn't that spoil it? I think it spoils it! (Middle-aged Female, PLHIV interview) 
Table 2

Health worker-reported barriers to and facilitators of use, completion and models of delivery for $3 \mathrm{HP}$ at Mulago ISS clinic in Uganda

\section{Potential Facilitators}

Common cause of mortality among stable PLHIV - In this clinic, of the 16,000 patients, most are stable and if you look at the cause of mortality it is TB related. ... So, it deserves much attention. (Medical Doctor at the clinic)

\section{Potential Barriers}

Inadequate understanding of TB and TPT- Some people think that TB is in a family, that it's hereditary. So, they will tell you, "You know my father had TB, so I also have TB or me I can't have TB in our family, no one has ever had TB". Such people will not adhere well on treatment. (Nurse at the clinic)

PLHIV trust their health workers - If you explain to them the benefits of taking this preventive treatment, they are usually receptive, and they usually take health workers information as kind of gospel truth. (Medical Doctor at the clinic)

Fear of TB- They think ...someone may die if they get the disease. They really have a great fear of the disease.... when they hear that there are preventive measures, everyone will want to take it to prevent them from not getting TB. (Nurse at the clinic)

Receptiveness to TPT- They seem to be eager to take the medicine including some requesting for it even though it was not prescribed for them .... They would say how come for me I haven't received? ....... So, when they hear of the preventive treatment, they will not hesitate to start the drugs. (Pharmacy Technician at the clinic)

Once-weekly 3HP dosing schedule- Taking it once a week rather than taking it daily much as the tablets are many. Maybe one will be like "anyway am taking many but once a week". (Medical Doctor at the clinic)

Streamlined clinic visits- If there is one specific staff, let me say nurse giving out this medicine it will help the patient and the clinic........ the patient will know where to go and not meander around. And even the health worker it will maximize the patient flow and the congestion in the clinic. (Nurse at the clinic)
Stigma associated with TB - We get challenges in trying to implement infection control procedures here because of the stigma associated with it even in the clinic. (Nurse at the clinic)

Fear of potential side effects- "Balese biragala kututta." (this literally means they have brought medications to kill us) ... And then other people think that the TB prevention medications, are very strong, they are very toxic that they will affect the liver and the kidney. (Nurse at the clinic)

Potential pill burden- Musawo (meaning health worker in the local language), I have been concentrating on my triple (three daily ARV pills) and now you want to give me more medication. ... some of them are not so positive about it. (Medical Doctor at the clinic)

Perceived challenges of DOT- Their work schedule I don't think it would allow them to come weekly..... most of our clients they are not self-employed, they are employed, and they have not disclosed....... So, it's very hard for someone to ask for permission every week the boss will get suspicious.... others travel long distances; others travel a lot. (Nurse at the clinic)

Perceived challenges of SAT- In DOT they take the medicine when you are really seeing, you can be sure that they are taking it. But for those ones taking it from home, sometimes they can take less, and you can't know. (Nurse at the clinic) 


\section{Potential Facilitators}

Perceived benefits of DOT- First, when this person comes weekly, the health worker will be able to see if there is any change; early detection of side effects that is one. And then second you are going to be sure this person has taken his or her medication. Because you are going to be there and see. (Nurse at the clinic)

Perceived benefits of SAT- First, the time you spend coming to the hospital, you save that time and transport. You would be doing some other things instead of coming to the hospital. And this person secures his or her job, yes. (Nurse at the clinic)

\section{Potential Barriers}

Technology challenges- Technology no, mostly we depend on self-report, pill count, say I have been taking so what is your balance? Then you negotiate around that. Technology no. (Nurse at the clinic) 
Table 3

Perceived facilitators and barriers to acceptance and completion of 3HP expressed as sources of behavior within the COM-B model

\section{Sources of Emergent themes behavior}

\section{PLHIV}

Health workers

\section{A. Facilitators}

Physical

capability

$\begin{array}{lll}\begin{array}{l}\text { Psychological } \\ \text { capability }\end{array} & \begin{array}{l}\text { Aware of being potentially } \\ \text { at risk of TB }\end{array} & \begin{array}{l}\text { Knowledge that TB causes the highest mortality } \\ \text { among PLHIV at the clinic }\end{array}\end{array}$

Awareness of easy

transmission of TB

$\begin{array}{ll}\begin{array}{l}\text { Physical } \\ \text { opportunity }\end{array} & \text { Perceived benefits of } \\ \text { DOT/SAT }\end{array}$

Perceived benefits of DOT/SAT

Social

opportunity

Reflective

Willingness to take TPT

PLHIV trust health workers

motivation

Trust in health workers

PLHIV are receptive to TPT

Once-weekly 3HP dosing schedule

Automatic

motivation

Fear of contracting TB

PLHIV fear TB

\section{B. Barriers}

Physical

capability

$\begin{array}{ll}\begin{array}{l}\text { Psychological Inadequate understanding } \\ \text { capability }\end{array} & \text { Inadequate understanding of TB and TPT }\end{array}$

Physical

opportunity

Perceived challenges of DOT/SAT

Technology challenges

Social

opportunity

Reflective

motivation

Automatic

motivation
Potential pill burden

Concerns about

effectiveness of $3 \mathrm{HP}$

Fear of potential side effects
Perceived challenges of DOT/SAT

Stigma associated with TB

Potential pill burden

Fear of potential side effects 
Table 4

Selected intervention functions for identified barriers to acceptance and completion of 3HP at Mulago ISS Clinic.

\begin{tabular}{|c|c|c|c|c|c|c|}
\hline \multicolumn{2}{|l|}{ Capability } & \multicolumn{2}{|l|}{ Opportunity } & \multicolumn{2}{|l|}{ Motivation } & \multirow{3}{*}{$\begin{array}{l}\text { Intervention } \\
\text { Functions }\end{array}$} \\
\hline Psychological & Physical & Physical & Social & Reflective & Automatic & \\
\hline $\begin{array}{l}\text { Inadequate } \\
\text { understanding } \\
\text { of TB \& TPT }\end{array}$ & $\begin{array}{l}\text { Health } \\
\text { worker } \\
\text { technology } \\
\text { challenges }\end{array}$ & $\begin{array}{l}\text { Perceived } \\
\text { challenges } \\
\text { of DOT \& } \\
\text { SAT }\end{array}$ & Stigma & $\begin{array}{l}\text { Pill burden, } \\
\text { effectiveness } \\
\text { concerns, } \\
\text { perceived } \\
\text { challenges } \\
\text { of DOT \& } \\
\text { SAT }\end{array}$ & $\begin{array}{l}\text { Fear of } \\
\text { side } \\
\text { effects }\end{array}$ & \\
\hline \multirow[t]{5}{*}{$\sqrt{ }$} & & & & $\sqrt{ }$ & & Education \\
\hline & & $\sqrt{ }$ & $\sqrt{ }$ & & & Enablement \\
\hline & & & & $\sqrt{ }$ & $\sqrt{ }$ & Persuasion \\
\hline & & $\sqrt{ }$ & & & & $\begin{array}{l}\text { Environmental } \\
\text { restructuring }\end{array}$ \\
\hline & $\sqrt{ }$ & $\sqrt{ }$ & & & & Training \\
\hline
\end{tabular}

\section{Supplementary Files}

This is a list of supplementary files associated with this preprint. Click to download.

- AdditionalFile1SRQR.docx 